\title{
sciendo
}

\section{THE ASSESSMENT OF GENERAL HEALTH STATUS IN PATIENTS WITH ODONTO-PERIODONTAL PATHOLOGY AND DIABETES MELLITUS}

\author{
ANDREEA DINU ${ }^{1}$, GEORGE MANIU ${ }^{2}$ \\ 1,2 "Lucian Blaga” University of Sibiu
}

Keywords: general health status, odonto-periodontal pathology, diabetes mellitus

\begin{abstract}
The present research attempts to assess the general health status of the studied group of patients by applying the questionnaire method. The research group has been composed of 210 subjects, from both urban and rural areas, having periodontal pathologies and disorders of the glucidic metabolism. In order to obtain information about the general health condition as well as the orodental hygiene, we have applied the questionnaire method, this being well individualized and adapted to be relevant to the present study. It was highlighted that patients with odonto-periodontal pathology and diabetes gave a more affected general health compared to those without diabetes.
\end{abstract}

\section{INTRODUCTION}

Diabetes mellitus (DM) is defined as a group of metabolic diseases characterized by hyperglycemia triggered by disorders of insulin secretion, insulin action or both.(1) Global prevalence of DM in 2014 was estimated at 9\% (2) and almost 1.6 billion deaths worldwide were directly attributed to DM in 2015.(3) DM is also associated to high morbidity due to a large range of complications such as retinopathy, nephropathy, neuropathy and cardiovascular diseases.(4,5) The prevention and management of these complications have become major aspects in modern treatment of diabetes.

Periodontal disease, one of the complications triggered by diabetes mellitus, is a chronic inflammatory condition. Several causes have been credited to the periodontal disease, both local and general. In this respect, there were incriminated tartar, functional disorders, morphologic changes of the teeth, of the dental arches and jaws, of the temporomandibular joint, occlusal and muscular, as well as general systemic factors. Currently, following extensive research, it is acknowledged the decisive role of the microbial factor in producing the periodontal disease in its destructive form, the other factors being favourable or predisposing. The determining factor in the destructive inflammatory diseases of the marginal periodontium is represented by the microbial factor, the bacterial plaque.(2)

The microbial etiology of periodontal disease is generally accepted nowadays, yet from more than 500 microbial species that are part of the bacterial plaque only a small fraction is involved in the pathogeny of periodontal disease.(4) Thus, the presence in the bacterial plaque of certain specific microorganisms able to produce substances with a destructive result for the periodontal tissues is one of the necessary conditions for the development of the periodontal disease.

The relationship between diabetes and periodontal diseases has been the main subject of more than 200 articles published in English language over the past 50 years. Up till now, diabetes is the only systemic condition obviously related to periodontal disease, through biochemical mechanisms

\section{AIM}

Obtaining data on the general and oral health status of patients with odonto-periodontal disease and diabetes mellitus compared to those without systemic pathologies by applying the questionnaire method. the method of applying the questionnaire.

\section{MATERIALS AND METHODS}

The study group consists of 210 subjects, who presented odonto-periodontal pathology and diabetes and who came in the dental office and in the University Dental Center of Sibiu between March 2018 - August 2020; the research included a witness group with the same number of subjects who were diabetes free.

The inclusion criteria for participation in the study of the subjects:

- The presence of clinical signs of periodontal disease:

- Gingival retraction;

- Gingival bleeding;

- Halitosis;

- Bone retraction, bone abscesses;

- Migration or dental mobility;

- $\quad$ The existence of an OPT radiography up to six months old;

- $\quad$ Subject age over 25 .

Exclusion criteria for participation in the study:

- Periodontal therapy in the last 12 months;

- Smokers;

- Unbalanced cardiovascular diseases;

- Respiratory or renal chronic diseases;

- Osteoporosis or rheumatoid arthritis;

- Pregnant or lactating women;

- Patients with incomplete data or who refuse participation in the study.

In order to assess the general health status, the subjects of the two groups participating in the research were asked to fill in a questionnaire. The applied questionnaire is based on a standard type and was adapted and individualized

${ }^{1}$ Corresponding author: Andreea Dinu, Str. Irina Rosetti, Nr. 18, Cisnădie, Cod 555330, E-mail: andreea.dinu@ulbsibiu.ro, Phone: +40753 919643 Article received on 20.06.2020 and accepted for publication on 27.08.2021 


\section{PUBLIC HEALTH AND MANAGEMENT}

through targeted questions about general health in order to be relevant to the present study. The questionnaire was numbered for each patient and each subject will keep the same number in all the files used in the following methods. The questionnaire contains confidential data, and that is why only the subjects' initials are used. The questions are concise and arranged in a logical order. The questionnaire regarding general data on the subjects' health status contains 48 questions of which 38 have four possible answers, while the rest have two possible answers. They are formulated as follows:

1. How is your general health status?

2. Compared to your health condition from 5 years ago, your current health status is?

3. Do you feel that you have weak hands or feet?

4. Do you feel numbness (tingling) in your hands or feet?

5. Do you have balance problems?

6. Do you feel that things no longer smell the same?

7. Do you feel that food no longer tastes the same?

8. Do you have difficulties in controlling your hand movements?

9. Do your hands tremble?

10. Do you have headaches?

11. Do you sweat for no reason?

12. Do you have nausea?

13. Do you have dizziness?

14. Do you feel shortness of breath without having exercised?

15. Do you have heart palpitations?

16. Do you hear noises or ringing in your ears?

17. Do you feel tired?

18. Do you feel hungry shortly after having eaten?

19. Do you have irritated eyes (redness, stinging, tearing)?

20. Do you have visual disorders?

21. Do you feel dizzy without drinking alcohol?

22. Do you have dry mouth or throat?

23. Do you have a sore throat (or cough sensation)?

24. Do you have a running nose?

25. Do you have a bad taste in your mouth?

26. Do you salivate more than usual?

27. Do you get easily irritated?

28. Do you have moments of depression?

29. Do you feel anxious or agitated?

30. Do you have trouble in managing your anger?

31. Do you have the tendency of forgetting things?

32. Do you have difficulty concentrating?

33. Do you have difficulty in reminding what you have read or seen on TV?

34. Do you tend to fall asleep at the wrong time?

35. Have you noticed a decrease in performance at work?

36. Have you noticed a loss of appetite?

37. Do you feel lack of energy?

38. Do you feel general fatigue?

39. Do you have difficulty in falling asleep?

40. Are you pregnant?

41. Do you smoke?

42. Do you suffer from cardiovascular disease?

43. Are you on medication for blood clotting?

44. Do you have respiratory problems?

45. Do you have digestive problems?

46. Are you on a special diet prescribed by a doctor?

47. Have you lost or gained more than $10 \mathrm{~kg}$ in the last few years?

48. Do you suffer from osteoporosis?

We presented the results as count and percentage. Differences between the two groups were assessed using ChiSquare or Fisher's exact test. Data analyses were performed using Core R Development Team software, Google sheets. P values $<0.05$ were considered statistically significant. (7-9)

\section{RESULTS AND DISCUSSIONS}

In both groups approximately $80 \%$ of the respondents consider that their health status is good, while over $95 \%$ from the control group and $83 \%$ of the witness group think that their current health status is approximately the same as 5 years ago.

Figure no. 1. Graphical analysis of health status by answering questions (group $\mathrm{C}$ or control group, group $\mathrm{M}$ or witness group)

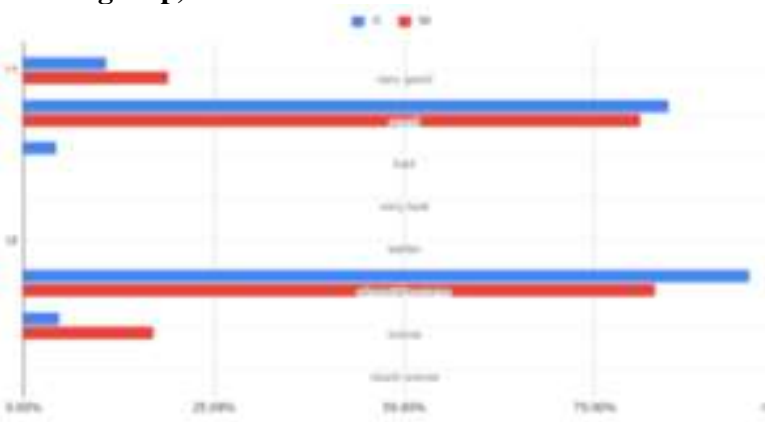

Significant differences between the two groups have been observed in the case of balance problems: approximately $40 \%$ of the subjects in the witness group have never had balance problems, as compared to the $20 \%$ subjects from the control group $(\mathrm{p}=0.000)$. The great majority from the control group (approximately 86\%) consider that they have changes in taste and smell as compared to those from the witness group, where the majority (approximately 70\%) claim they have never or rarely experienced these changes. $(\mathrm{p}=0.000)$

Figure no. 2. Graphic analysis of the data concerning the health status following the answers to questions I 3-8 (group C or control group, group $\mathrm{M}$ or witness group)

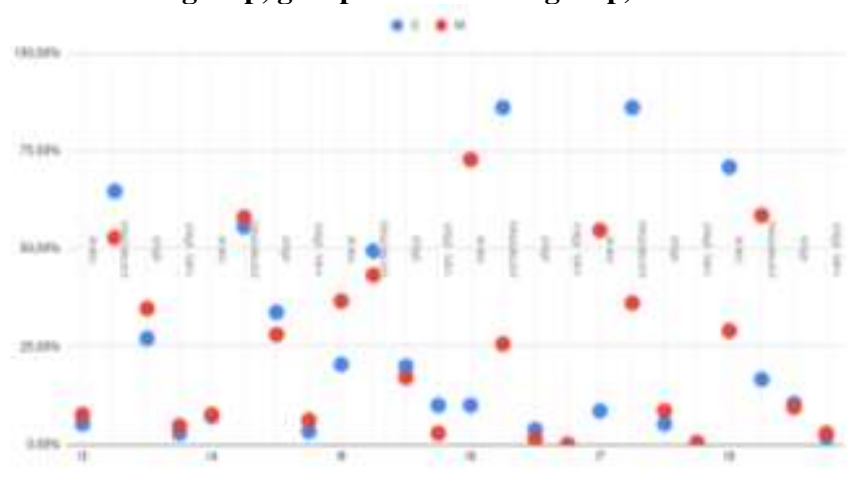

Figure no. 3. Graphic analysis of the data concerning health status following the answers to questions I 9 - 14 (group C or control group, group $\mathrm{M}$ or witness group)

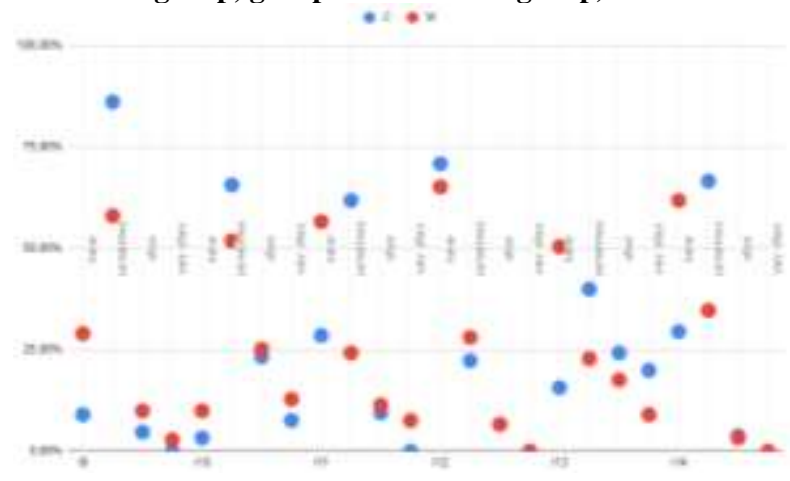




\section{PUBLIC HEALTH AND MANAGEMENT}

As for the occurrence of headaches, $65.71 \%$ of the witness group claim they rarely have these conditions, while $86.19 \%$ assert they experience trembling hands, and $29.05 \%$ declare that they have never had such conditions $(\mathrm{p}=0.000)$. $61,90 \%$ of the control group declare that they sweat with no reason, while $40 \%$ state they had experienced dizziness. However, $50 \%$ from the witness group claim they do not have these conditions $(\mathrm{p}=0.000)$

The vast majority of the subjects in the control group claim they hear noises in their ears $(70 \%)$, they feel fatigued $(54.29 \%)$, they feel intensively hungry shortly after having eaten $(49.52 \%)$, while approximately half of the subjects in the witness group answered they have never had such symptoms $(p=0.000)$. Also, $63.81 \%$ of the control group claim they have $(\mathrm{p}=0.000)$

Figure no. 4. Graphic analysis of the data concerning the health status following the answers to questions I $15-20$ (group $\mathrm{C}$ or control group, group $\mathrm{M}$ or witness group)

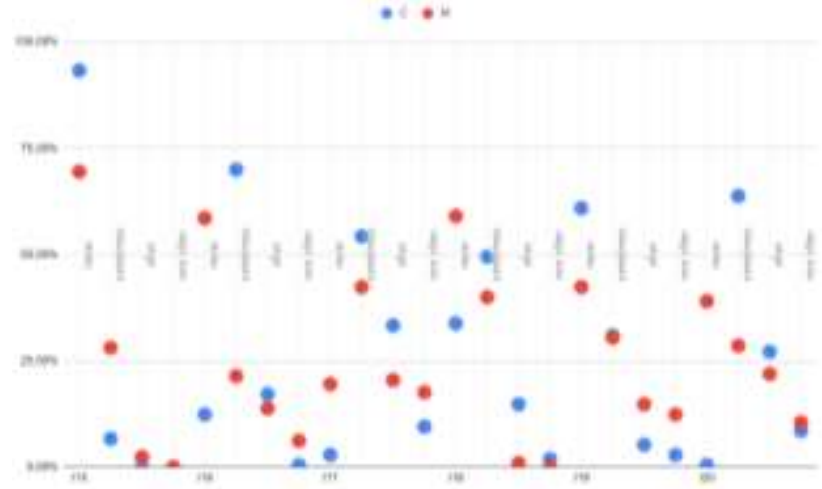

The significant differences between the two groups were observed in the case of causeless dizziness, dry or sore throat, xerostomia, presence of nasal secretions, bad taste in the mouth, where the vast majority of the control group (approximately 60\%) claim they sometimes experience such symptoms, while subjects in the witness group state they have never had such symptoms $(\mathrm{p}=0.000)$

Figure no. 5. Graphic analysis of data concerning health status by answering the (group $\mathrm{C}$ or control group, group $\mathrm{M}$ or witness group)

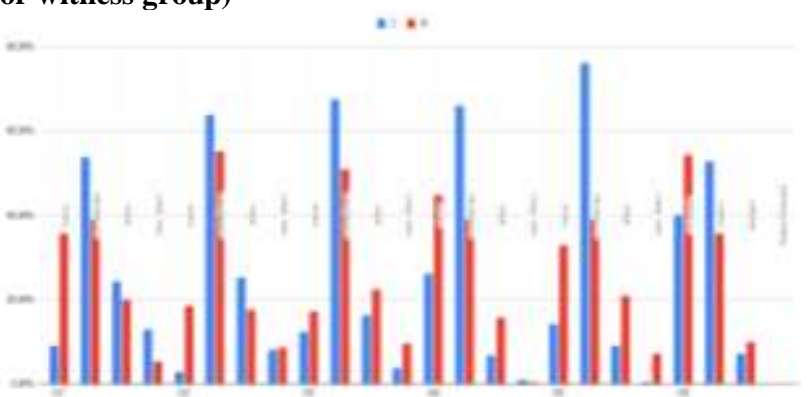

More than half of the subjects in the control group state they sometimes are jumpy, depressive, anxious or agitated, and have difficulty with anger management, while those in the witness group declare they had no such problems $(\mathrm{p}=0.000)$. $17.62 \%$ in the control group frequently forget, while $26.67 \%$ in the witness group state they do not have this problem $(\mathrm{p}=0.000)$.

\section{DISCUSSIONS}

In the specialized literature, the connections between the general and the oral status have been proven in numerous studies, these being reflected by the term "oral-systemic link".

It has been shown that chronic inflammation, even of low intensity, can contribute to the occurrence of secondary systemic diseases. As chronic periodontitis is an inflammatory disease, it can be associated with an inflammatory reaction at the systemic level in various organs. Although the pathophysiological mechanisms are not yet fully known, there has been evidence of an association between periodontitis and inflammation that occurs at the systemic level, with diseases such as cardiovascular disease, diabetes, respiratory disease, kidney disease, rheumatoid arthritis, malignancies.(10)

The association between diabetes and periodontal disease is considered to be bidirectional, an elevated blood glucose level promotes the development of oral germs, and periodontal disease associated with systemic inflammation may contribute to exacerbation of insulin resistance.

There are close correlations between general and oral status that are also manifested in relation to age and socioeconomic status and with an impact on quality of life. Numerous studies in the literature indicate that the main strategy to prevent the onset and evolution of periodontal disease is to control inflammation.

\section{CONCLUSIONS}

The analysis of the data collected following the application of the questionnaire shows that the addressability to family medicine and dentistry services is low, the results being similar in both the control group and the witness group. It was highlighted that patients with odonto-periodontal pathology and diabetes gave a more affected general health compared to those without diabetes.

Diabetes is known to be a disturbingly prevalent condition. We believe that the prevention of diabetes mellitus could help slow the spread of this disease, while the prevention and control of periodontal disease should be considered an integral part of diabetes control.

\section{REFERENCES}

1. Rohani B. Oral manifestations in patients with diabetes mellitus. World Journal of Diabetes. 2019 Sep 15;10(9):485.

2. Rajhans NS, Kohad RM, Chaudhari VG, Mhaske NH., A clinical study of the relationship between diabetes mellitus and periodontal disease., J Indian Soc Periodontol. 2011 Oct;15(4):388-92.

3. Preshaw PM, Alba AL, Herrera D, Jepsen S, et al, Periodontitis and diabetes: a two-way relationship, Diabetologia. 2012;55:21-31.

4. Lamster IB, Lalla E, Borgnakke WS, Taylor GW. The relationship between oral health and diabetes mellitus, J Am Dent Assoc. 2008;139:19S-24S.

5. Dumitriu HT. Parodontologie. Editura Viaţa Medicală Românească, ediţia a V-a, Bucureşti, 2009

6. American Diabetes Association. Standards of Medical Care in Diabetes. In Diabetes Care, vol.34, supplement 1, January 2011.

7. R Development Team. R: A Language and Environment for Statistical Computing. R Foundation for Statistical Computing; 2009.

8. Mocan I. SPSS Introduction in data analysis; Lucian Blaga University Publishing House: Sibiu, Romania; 2005.

9. Maniu I. Data analysis techniques: statistics; Lucian Blaga University Publishing House: Sibiu, Romania; 2014.

10. Kumar PS. From focal sepsis to periodontal medicine: A century of exploring the role of the oral microbiome in systemic disease. J Physiol. 2017 Jan 15;595(2):465-476. 